\title{
Fluctuations in Saffman-Taylor fingers with quenched disorder
}

\author{
M. Torralba, ${ }^{1}$ J. Ortín, ${ }^{1}$ A. Hernández-Machado, ${ }^{1}$ and E. Corvera Poiré ${ }^{1,2, *}$ \\ ${ }^{1}$ Departament ECM, Facultat de Física, Universitat de Barcelona, Diagonal 647, E-08028 Barcelona, Spain \\ ${ }^{2}$ Departamento de Física y Química Teórica, Facultad de Química, UNAM Ciudad Universitaria, México D.F. 04510, México
}

(Received 17 November 2005; published 3 April 2006)

\begin{abstract}
We make an experimental characterization of the effect that static disorder has on the shape of a normal Saffman-Taylor finger. We find that static noise induces a small amplitude and long wavelength instability on the sides of the finger. Fluctuations on the finger sides have a dominant wavelength, indicating that the system acts as a selective amplifier of static noise. The dominant wavelength does not seem to be very sensitive to the intensity of static noise present in the system. On the other hand, at a given flow rate, rms fluctuations of the finger width, decrease with decreasing intensity of static noise. This might explain why the sides of the fingers are flat for typical Saffman-Taylor experiments. Comparison with previous numerical studies of the effect that temporal noise has on the Saffman-Taylor finger, leads to conclude that the effect of temporal noise and static noise are similar. The behavior of fluctuations of the finger width found in our experiments, is qualitatively similar to one recently reported, in the sense that, the magnitude of the width fluctuations decays as a power law of the capillary number, at low flow rates, and increases with capillary number for larger flow rates.
\end{abstract}

DOI: 10.1103/PhysRevE.73.046302

PACS number(s): 47.54. $-\mathrm{r}, 05.10 .-\mathrm{a}, 47.56+\mathrm{r}$

\section{INTRODUCTION}

The growth of interfaces in disorder media has been studied extensively in the case of rough, macroscopically stable interfaces both experimentally and theoretically [1]. However, the effect of disorder media on unstable interfaces has not been so well explored [2]. A prototypical example of unstable interface is the Saffman-Taylor finger [3-5] and our aim in this paper is the experimental characterization of the effect of quenched noise on it. A Saffman-Taylor finger is the steady state pattern that is formed between two fluids when a low viscosity fluid displaces a high viscosity fluid in a rectangular Hele-Shaw cell. In a classical experiment and in the absence of perturbations, the finger width is greater than half of the channel width and the finger is called normal. In the presence of large perturbations or in anisotropic cases, the width of the finger can be less than half of the channel width and the finger is called anomalous. In the closely related problem of crystal growth, when an undercooled melt solidifies, the solid front has a parabolic shape and lateral protrusions called side branches. These ones grow in amplitude as they are advected away from the parabolic tip. This phenomenon has been widely investigated in solidification both, experimentally $[6,7]$ and theoretically $[8-10]$ with the conclusion that side branching results from selective amplification of natural noise. In anomalous Saffman-Taylor fingers, dendrites have been observed using localized disturbances such as a bubble placed at the finger tip or a thread placed along the channel $[11,12]$. Normal Saffman-Taylor fingers are characterized by a stable curved front and flat sides. However, noise unintentionally present in the system, is not enough to create side branching in typical Saffman-Taylor fingers.

Recently, two situations in which normal Saffman-Taylor fingers do not have flat sides have been reported $[13,14]$.

\footnotetext{
*Author to whom correspondence should be addressed. Electronic address: eugenia.corvera@gmail.com
}

Experimental fluctuations in Saffman-Taylor fingers were reported for systems with very large aspect ratios $W / b$ [13], where $W$ is the cell width and $b$ is the gap spacing between the plates. It is observed that for the larger aspect ratios studied fluctuations on the finger width are visible for all flow rates. Moreover, for the smaller aspect ratios studied, fingers appear to be steady as in the classic Saffman-Taylor steady-state case, but with sufficient resolution, fluctuations on the finger width could be measured for all flow rates up to the onset of finger instability. A key point in this work is that the magnitude of the width fluctuations at low velocities decays as a power law of the capillary number $\mathrm{Ca} \equiv \eta U / \sigma$ with a power of approximately $-2 / 3$. Where $\eta$ is the dynamic viscosity, $U$ is the finger velocity and $\sigma$ is the surface tension. This fluctuation power law remained unchanged for all their experimental variations. In a different context, a dynamically induced, low amplitude, long wavelength, lateral instability of the Saffman-Taylor finger was predicted [14] by means of numerical integration of a phase field model [15]. Such an instability was observed in two situations in which small dynamic perturbations were overimposed to a constant pressure drop. For the first situation, the dynamic perturbation consisted of an oscillatory mode. This gave rise to a strictly periodic undulation of the finger sides. In the second situation, the dynamic perturbation consisted of temporal noise. This one induced a nonperiodic lateral instability. For both situations the instability undergoes a process of mode selection and the results seem to indicate that the system acts as a selective noise amplifier.

In the present work, we characterize the effect that quenched (or static) disorder has on the normal SaffmanTaylor finger shape. For this purpose, we intentionally chose cells with small aspect ratios $W / b$ that in the absence of quenched disorder, would lead to normal Saffman-Taylor finger shapes with flat sides. We find fingers that present a lateral instability with a dominant wavelength in agreement with Ref. [14]. This seems to indicate that the system acts as a selective amplifier of quenched noise. On the other hand, 
when we measure rms fluctuations of the finger width as a function of the capillary number we find results that are qualitatively similar to those of Ref. [13] in the sense that the magnitude of the width fluctuations at low capillary number decays as a power law of $\mathrm{Ca}$, and increases for large values of it.

\section{EXPERIMENTAL SETUP AND DATA ANALYSIS}

The experiments were performed in a rectangular HeleShaw cell formed by two horizontal glass plates of thickness $19 \mathrm{~mm}$, separated by a narrow gap spacing. In order to introduce quenched disorder in the system, an auxiliary fiber glass plate of thickness $1.7 \mathrm{~mm}$ with copper islands was introduced between the two glass plates, in such a way that the fluid flow takes place between the fiber glass plate and the upper glass plate. This fiber glass plate was fixed on the bottom glass plate. The copper islands were squares of dimensions $1.5 \mathrm{~mm} \times 1.5 \mathrm{~mm}$ and height $h=0.06 \mathrm{~mm}$ and occupied random sites of a square grid. The plates used in our experiments are described in Ref. [16]. Two different kinds of plates were used in the experiments: low occupation ratio (10\% of the total area filled with copper islands) and high occupation ratio (35\% of the total area filled with copper islands) which for convenience will be called, in the rest of the paper, low and high coverage, respectively. The width of the channel $W$ was fixed to $24 \mathrm{~mm}$. The gap spacings (measured as the distance between the fiber glass plate and the upper glass plate) in the reported experiments were $b=0.8 \mathrm{~mm}$ and $b=1.3 \mathrm{~mm}$. This implies relative variations in the gap spacing of $7.5 \%$ and $4.6 \%$, respectively. The experiments were performed with different disorder plates: four disorder plates in the experiments with $b=1.3 \mathrm{~mm}$ and $10 \%$ coverage and three disorder plates in the other experiments. The cell was filled with silicone oil Rhodorsil $47 \mathrm{~V}$ with a dynamic viscosity $\eta=0.5 \mathrm{Pas}$ and surface tension $\sigma=20.7 \mathrm{mN} / \mathrm{m}$. The silicone oil was colored with Oil Blue $\mathrm{N}$ (Aldrich). Rheological measurements showed that changes in viscosity due to coloring were below $2 \%$. In order to form fingers, the oil in the cell was withdrawn from one end of the channel at constant flow rate using a syringe pump. The other end of the channel was left open for air to penetrate freely. The velocity range of the fingers in the experiments reported was $(0.11,15) \mathrm{mm} / \mathrm{s}$.

Pictures of the finger were obtained with a CCD camera mounted on top of the channel, which was illuminated from below. The camera could travel along the cell at velocities in the range $(-20,20) \mathrm{mm} / \mathrm{s}$. The frames captured with the CCD had a fixed size of $640 \times 480$ pixels. Since the amplitude of the fluctuations in finger width was relatively small, the size of the pixel was fixed to $0.077 \mathrm{~mm} /$ pixel in order to have good resolution. To obtain the pictures of the fingers analyzed, the CCD camera was placed at the end of the cell open to air, and a finger was generated. When the finger tip had advanced a distance much larger than the channel width, the CCD traveled towards the finger tip at a high velocity while acquiring images at two frames per second. By mounting several frames, the complete image of very long fingers (see Fig. 1) was obtained.

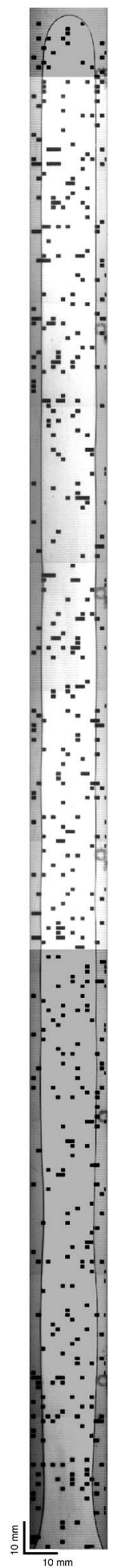

FIG. 1. Finger in a cell with $10 \%$ coverage and gap spacing $b$ equal to $b=0.8 \mathrm{~mm}$. The velocity of the finger is $U=2 \mathrm{~mm} / \mathrm{s}$. The bright region is defined as the finger body, that is, the portion of the finger for which the amplitude of the instability has saturated. The dark regions are the tail and tip of the finger. The tail of the finger experiences an instability whose amplitude changes in the course of the whole experiment. 
In the long fingers generated in our experiments, three different regions could be detected, as shown in Fig. 1: tip, body, and tail. The tip is the front part of the propagating finger where the lateral instability has not reached its full amplitude. The tail of the finger is the region that stays far away from the propagating finger tip. For this region, the amplitude of the fluctuations does not saturate. That is, as the finger advances, the tail width is not constant in time. Last, we observe that for the central part of the finger, which we call body, the amplitude of the instability saturates, and its shape, in the laboratory frame of reference, is constant in time. For this region pictures of the finger at different times are therefore identical. In order to determine the size of the tip region, pictures of tips at different times were superimposed to each other. For the body and tail regions, we have left the camera fixed in space and have taken pictures during the course of the whole experiment. Once the pictures are overimposed, it is possible to determine the spatial extent of each region. The size of the tail was found to be almost independent of the velocity of the finger, and the size of the tip did show a nonmonotonic dependence with velocity in agreement with the rest of the quantities reported. Care was taken to distinguish the three regions in each case and the results presented in this paper correspond exclusively to the finger body.

Two types of analysis were done: frequency distributions of the lateral instability and fluctuations of the finger width. For the frequency distribution analysis, we associate a frequency to a distance through the relation

$$
\nu=\frac{U}{\Lambda},
$$

where $U$ is the average finger velocity, and $\Lambda$ is the distance between pairs of adjacent maxima that appear on a given side of the finger. Measurements on both sides of the finger were considered independently since fluctuations on both sides of it do not seem to be correlated in an obvious way. This type of analysis was used in Ref. [14]. On the other hand, fluctuations of finger width were characterized by the rms fluctuations of the finger width along the finger. That is, we have defined the rms fluctuations of the finger width as $\delta_{\lambda}=\sqrt{ }\left[\left\langle(\lambda-\langle\lambda\rangle)^{2}\right\rangle\right]$ where the averages mean spatial variations over the body of the finger, as defined above. It is important to note that our fluctuations are not measured as the ones in Ref. [13], since we are measuring fluctuations of the finger width in space rather than fluctuations of the finger width in time. Since rms fluctuations of the finger width are a measure of the amplitude of the lateral instability, the larger the value of $\delta_{\lambda}$, the more easily the lateral instability can be observed.

\section{NATURAL FREQUENCIES}

Before describing the experimental results of the present paper, it is convenient to remember that there are two natural frequencies of the steady-state problem. The first characteristic frequency is the finger velocity $U$ divided by the finger width $\lambda W$, which gives a frequency equal to

$$
\nu_{f}=\frac{U}{\lambda W} .
$$

The other frequency characteristic of the steady-state problem is the one determined by the flow very far from the finger tip, that is, the flow velocity at infinity $V_{\infty}$ divided by the channel width $W$, which gives a frequency equal to

$$
\nu_{\infty}=\frac{V_{\infty}}{W} .
$$

These two frequencies are related to each other since conservation of matter implies that $U \lambda=V_{\infty}$. Therefore, we can write the characteristic frequency of the finger in terms of the characteristic frequency at infinity as

$$
\nu_{f}=\frac{1}{\lambda^{2}} \nu_{\infty} .
$$

For our experiments, fingers do not have a constant width. Therefore, in order to have reference frequencies for the analysis, we keep expressions (3) and (4) for the characteristic frequencies of the system, where the meaning of $\lambda W$ is now the average finger width.

\section{RESULTS}

We have chosen only experimental configurations for which static noise is low enough to preserve a single finger propagating into the cell. The tip of these fingers resembles a normal Saffman-Taylor finger and it is stable in the presence of quenched noise. On the other hand, the flat sides of the finger are unstable. As a result, a lateral instability grows and gives rise to a low amplitude and long wavelength structure reminiscent of incipient dendrites. As the finger propagates, the instability propagates away from the tip. During this propagation, the amplitude of the instability grows until it reaches a maximum amplitude. After this happens, in the laboratory frame of reference, for the body of the finger, the shape of the finger sides is stationary. This stationary shape is reproducible for a given configuration of static disorder. Clearly, the details of the fluctuations on the finger sides differ for different configurations of static disorder.

\section{A. Variation of static disorder}

Frequency distributions for three geometries are shown in Fig. 2. In all cases the flow rate has been chosen in order to have, up to two significant figures, equal finger velocities for the three geometries. The distributions are results of the different configurations of static disorder for each geometry. Experimental parameters for the three cases are shown in Table I. Fingers representative of each of the three geometries are illustrated in Fig. 3. There are two variables that may change the properties of static disorder in the cell: the coverage and the gap spacing.

The first way in which we study the dependence of the lateral instability with quenched disorder is by keeping constant the coverage and varying the gap spacing. The smallest the gap, the larger the intensity of static noise present in the system. In the top and center graphs of Fig. 2, we see fre- 


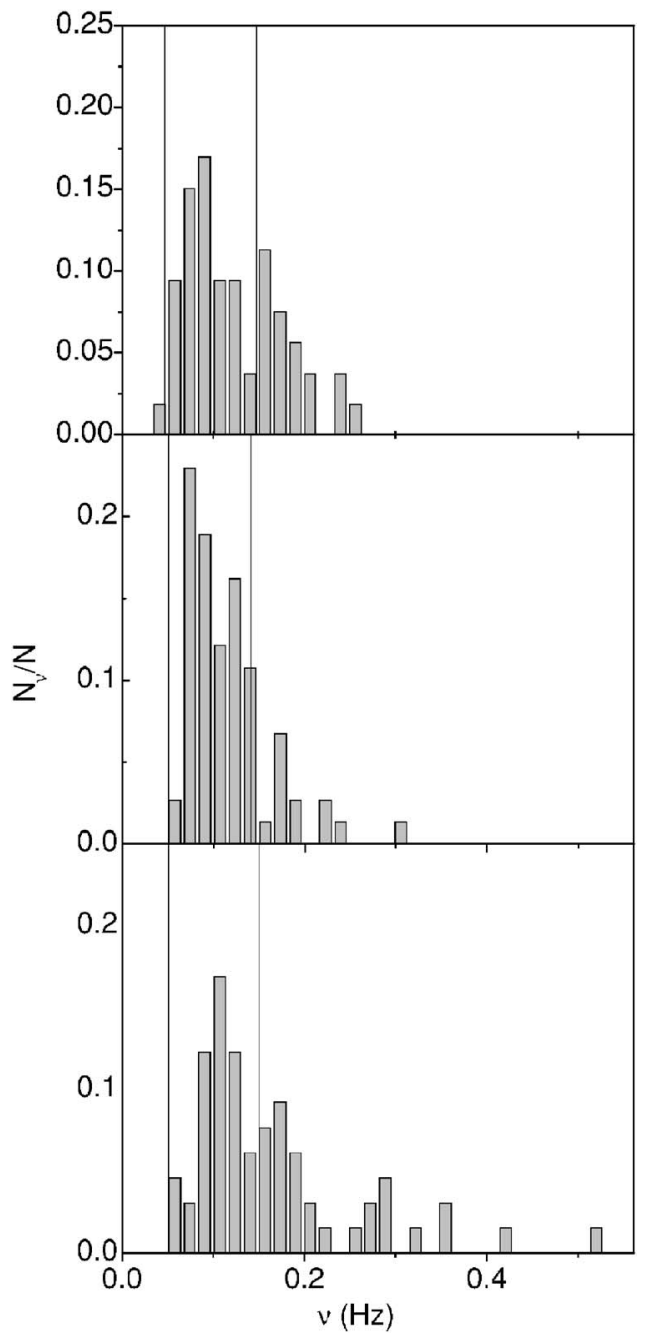

FIG. 2. Distributions of frequencies of the lateral instability corresponding to the three experimental configurations of Fig. 3. Vertical lines are the characteristic frequencies of the steady state $\left[\nu_{f}\right.$ at the right and $\nu_{\infty}$ at the left, as defined in Eqs. (2) and (3)]. From top to bottom, $b=0.8 \mathrm{~mm}$ and $10 \%$ coverage, $b=1.3 \mathrm{~mm}$ and $10 \%$ coverage, $b=1.3 \mathrm{~mm}$ and $35 \%$ coverage.

quency distributions for gap spacings of $0.8 \mathrm{~mm}$ and $1.3 \mathrm{~mm}$, corresponding to relative variations in the gap of $7.5 \%$ and $4.6 \%$, respectively. The coverage is equal to $10 \%$. In both cases, the distributions have peaks that are between the two characteristic frequencies of the system and the

TABLE I. Experimental parameters of the three geometries studied. $b$ is the effective gap, $W$ is the cell width, $h / b$ is the relative variation in gap spacing, $\nu_{\infty}$ is the characteristic frequency at infinity.

\begin{tabular}{lccc}
\hline \hline$\%$ Coverage & 10 & 10 & 35 \\
\hline$b(\mathrm{~mm})$ & 0.8 & 1.3 & 1.3 \\
$W(\mathrm{~mm})$ & 24 & 24 & 24 \\
$\% h / b$ & 7.5 & 4.6 & 4.6 \\
$V_{\infty}(\mathrm{mm} / \mathrm{s})$ & 1.12 & 1.22 & 1.22 \\
$\nu_{\infty}(\mathrm{Hz})$ & 0.047 & 0.051 & 0.051 \\
\hline \hline
\end{tabular}

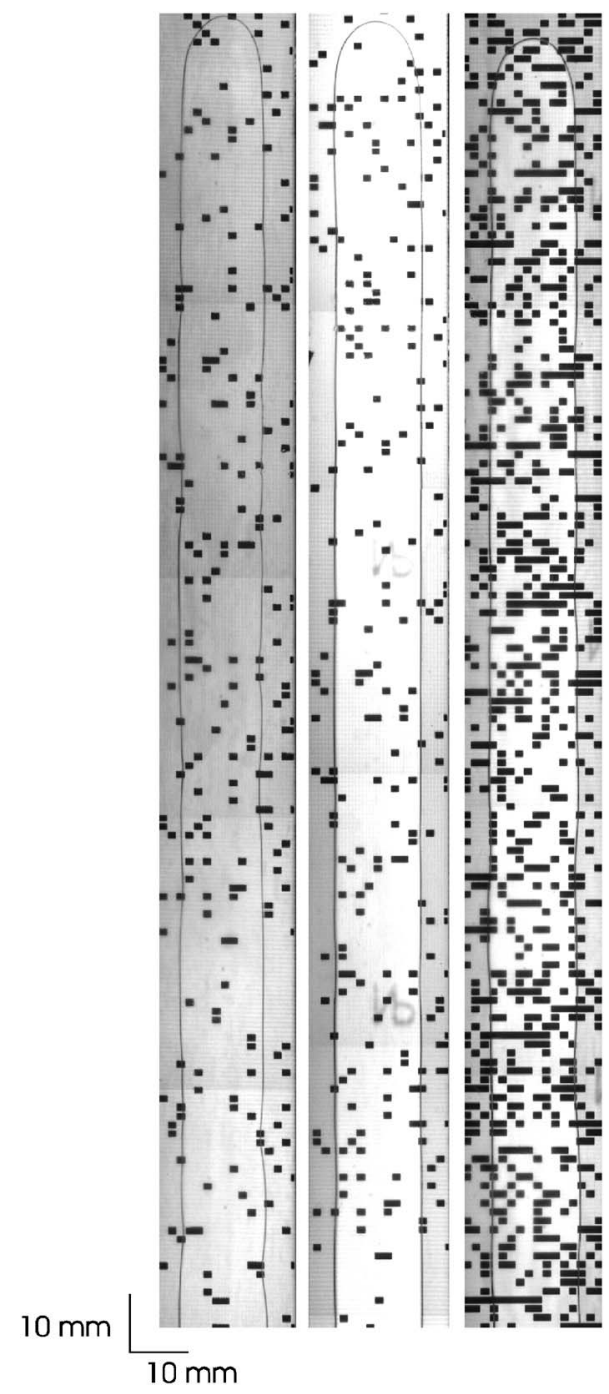

FIG. 3. Fingers in the presence of different configurations of quenched disorder. For all of them, the finger velocity is $U=2 \mathrm{~mm} / \mathrm{s}$. From left to right, $b=0.8 \mathrm{~mm}$ and $10 \%$ coverage, $b=1.3 \mathrm{~mm}$ and $10 \%$ coverage, $b=1.3 \mathrm{~mm}$ and $35 \%$ coverage. Only finger tips and bodies are shown.

smallest frequencies observed are close to the characteristic frequency of infinity. Also, both have tails that decay at frequencies few times larger than the characteristic frequency of the finger. There is no appreciable change in the width of the distributions. The standard deviations of the data, $0.052 \mathrm{~Hz}$ and $0.046 \mathrm{~Hz}$ for the $0.8 \mathrm{~mm}$ and $1.3 \mathrm{~mm}$ gaps, respectively, and the means of the distributions, $0.124 \mathrm{~Hz}$ and $0.116 \mathrm{~Hz}$ for the $0.8 \mathrm{~mm}$ and $1.3 \mathrm{~mm}$ gaps, respectively, also indicate that there is not an obviously quantifiable difference in the distributions.

The second way in which we change the properties of the static disorder in the cell is by keeping constant the gap spacing and varying the coverage. In the center and bottom graphs of Fig. 2 we see the frequency distributions for coverages of $10 \%$ and $35 \%$. The gap spacing is equal to $1.3 \mathrm{~mm}$ in both cases, the distributions have peaks that are between the two characteristic frequencies of the system and the smallest frequencies observed are close to the characteristic 
TABLE II. Finger characteristics for the three geometries studied. $U$ is the average finger velocity, $\lambda$ is the average finger width in units of the channel width $W, \nu_{f}$ is the characteristic frequency of the finger. $N$ is the total number of measurements of $\Lambda$ for each geometry.

\begin{tabular}{llll}
\hline \hline \% Coverage & 10 & 10 & 35 \\
\hline$b(\mathrm{~mm})$ & 0.8 & 1.3 & 1.3 \\
$U(\mathrm{~mm} / \mathrm{s})$ & 2.01 & 2.03 & 2.01 \\
$\lambda$ & 0.56 & 0.60 & 0.58 \\
$\nu_{f}(\mathrm{~Hz})$ & 0.147 & 0.141 & 0.150 \\
$\delta_{\lambda}(\mathrm{mm})$ & 0.0185 & 0.0064 & 0.01146 \\
$N$ & 53 & 74 & 65 \\
\hline \hline
\end{tabular}

frequency of infinity. The tail for high coverage falls to somewhat larger frequencies than the tail for small coverage. This indicates that high coverage induces the presence of fluctuations of small wavelength in the lateral instability. Nevertheless, in both cases, the tail of the distribution decays at frequencies few times larger than the characteristic frequency of the finger. The standard deviations of the data, $0.046 \mathrm{~Hz}$ and $0.090 \mathrm{~Hz}$ for coverages of $10 \%$ and $35 \%$, respectively, and the means of the distributions, $0.116 \mathrm{~Hz}$ and $0.164 \mathrm{~Hz}$ also indicate that the range of frequencies that grow in the lateral instability, is somewhat larger for the high coverage case and that the distribution is slightly shifted towards high frequencies which correspond to short wavelengths.

Table II shows average finger velocities, average finger widths, frequencies characteristic of the finger and rms fluctuations of the finger width corresponding to the same experiments of Fig. 2 and Table I. From the rms fluctuations of the finger width, it is clear that fluctuations increase for a smaller gap at constant coverage (compare first and second columns of the last row in Table II) and increase for larger coverage at constant gap (compare second and third columns of the last row in Table II). Since the rms fluctuations of the finger width are a measure of the amplitude of the lateral instability, it becomes clear that fluctuations increase with the two possible ways of increasing the intensity of static noise in the cell.

Summarizing, for the three geometries with static disorder discussed, the frequency distribution of the lateral instability has a peak that lies between the two characteristic frequencies of the steady state. This seems to imply that, for a given velocity, there is a preferred frequency for the instability. In other words, the system is acting as a selective amplifier of static noise. The distribution does not seem to be very sensitive to how much static noise is present in the system. Moreover, rms fluctuations of the finger width increase with increasing intensity of static noise.

\section{B. Variation with capillary number}

We have studied the finger instability with static disorder for different flow rates. As mentioned in the introduction, we have intentionally chosen to work with cells of low aspect

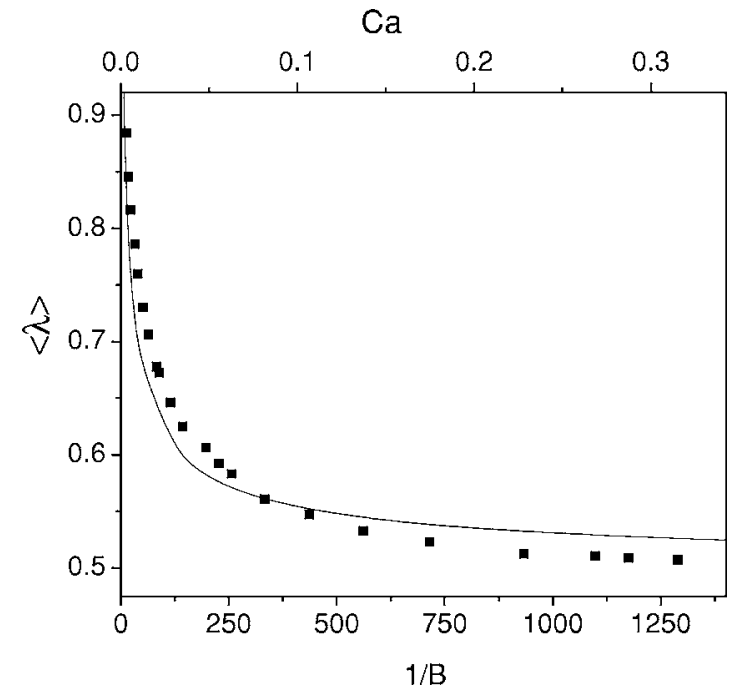

FIG. 4. Mean finger width as a function of $1 / B \equiv 12(W / b)^{2} \mathrm{Ca}$. The continuous line is the theoretical McLean and Saffman curve [17]. Solid squares are the experimental values of the mean width averaged over four different disorder configurations. As can be seen, our experiments give, in all cases, normal Saffman-Taylor fingers, that is, fingers whose width is larger than half of the channel width.

ratio $W / b$. The purpose of this was, on the one hand, to have fingers that in the absence of quenched disorder would have flat sides and, on the other, to have normal fingers, that is, fingers whose width was larger than one-half of a channel width. A plot of the average finger width as a function of capillary number (Fig. 4), shows that, for all of our experiments, we have normal Saffman-Taylor fingers. Agreement with the McLean and Saffman curve is similar to results for the steady state reported in literature.

\section{Fluctuations of the finger width}

We have studied how the lateral fluctuations of the finger change with capillary number. For this purpose, we have done an analysis similar to the one carried out in Ref. [13], but measuring spatial variations instead of temporal variations of the finger width as explained in Sec. II. Figure 5 shows how fluctuations change with capillary number. We have obtained results that are qualitatively similar to those of Ref. [13] in the sense that rms fluctuations of the finger width decrease with increasing $\mathrm{Ca}$ for small values of it and scale as a power law. Also, at larger values of $\mathrm{Ca}$, rms fluctuations of the finger width increase with increasing $\mathrm{Ca}$. Figure 6 shows typical fingers for six values of $\mathrm{Ca}$ indicated in Fig. 5. Fingers appear compressed in the horizontal axis, in order to facilitate the visual appreciation of the fluctuations. From the figures, it is clear the nonmonotonic size of the rms fluctuations as $\mathrm{Ca}$ increases. Moreover, our results for the rms fluctuations of the finger width in the presence of static disorder appear to scale, at low $\mathrm{Ca}$, as the power law $\mathrm{Ca}^{-1 / 3}$.

\section{Frequency distributions of the lateral instability}

In order to study the modes that compose the lateral fluctuations, we have computed, as in Sec. IV A, frequency dis- 


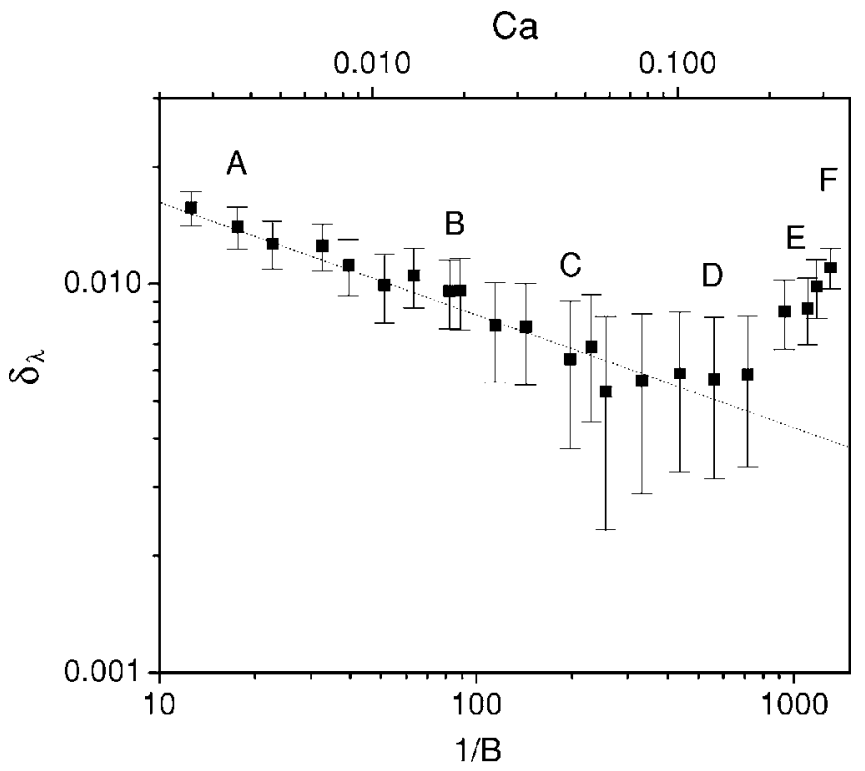

FIG. 5. Fluctuations in the finger width vs $1 / B$. Solid squares are the average fluctuations over four different disorder configurations. Dotted line is the best fit for the low velocity regime $(1 / B$ from 0 to 250$): \delta_{\lambda}=(0.034 \pm 0.008)(1 / B)^{(-0.30 \pm 0.13)}$. Letters correspond to capillary numbers for which a finger is illustrated in Fig. 6.

tributions. Figure 7 shows frequency distributions for different velocities. We have also plotted the variations of the mean and standard deviations for the data contained in the distributions as a function of $\mathrm{Ca}$. We can see this in Fig. 8. From both figures, it is clear that the frequency that dominates the pattern of the lateral fluctuation increases with increasing $\mathrm{Ca}$ indicating that at large $\mathrm{Ca}$, short wavelength fluctuations are more and more favorable. Also, the width of the distribution increases with increasing $\mathrm{Ca}$ indicating that the range of frequencies that are not suppressed by surface tension, increases with increasing $\mathrm{Ca}$. This is in qualitative agreement with the primary instability that gives rise to viscous fingers in the first place, that is, the one that destabilizes the flat interface. From Fig. 8 we can see that the mean frequency increases almost linearly with $\mathrm{Ca}$.

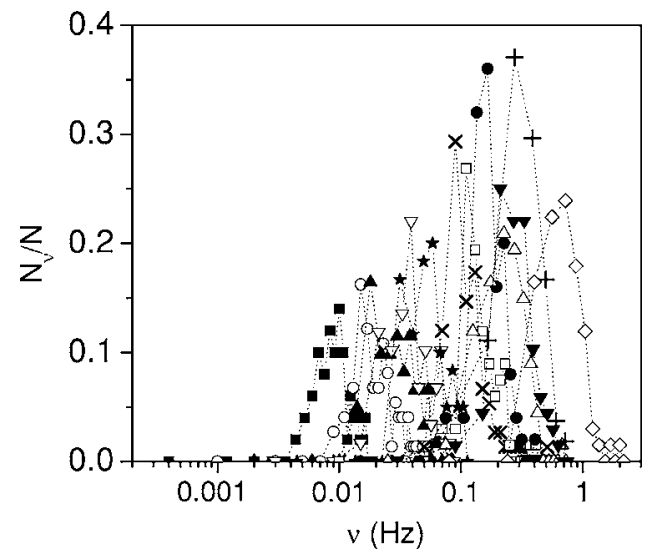

FIG. 7. Distributions of frequencies of the lateral fluctuations at different velocities. The symbols are $\mathbf{\square}, 1 / B=23 ; \circ, 1 / B=39 ; \boldsymbol{\Delta}$, $1 / B=63 ; \nabla, 1 / B=82 ; \star, 1 / B=118 ; \times, 1 / B=198 ; \square, 1 / B=254 ; \bullet$, $1 / B=339 ; \quad \triangle, \quad 1 / B=435 ; \quad \nabla, \quad 1 / B=558 ; \quad+, 1 / B=925 ; \quad \diamond$, $1 / B=1268$.

We have made a linear rescaling of frequencies in order to study the possible collapse of the distributions. For this purpose, we have mapped to zero the frequency at infinity and to one the frequency of the finger. We observe an apparent collapse of the distributions with means that lie all between zero and one and no particular tendency as a function of capillary number. Nevertheless, the standard deviation seems to have a tendency to decrease with increasing $\mathrm{Ca}$, indicating that there is no real collapse of the distributions.

\section{DISCUSSION}

Static noise induces an instability on the sides of the Saffman-Taylor finger. The instability generates small amplitude and long wavelength fluctuations that undergo a process of selection. That is, modes grow and compete dynamically, and the competition gives rise to a nonperiodic structure whose frequency distribution has a well characterized peak. Such a peak lies between the two characteristic frequencies (a)

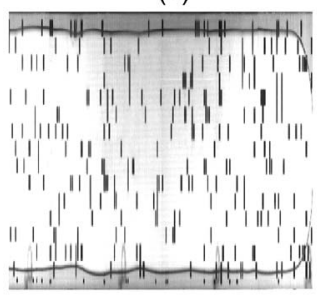

(d)

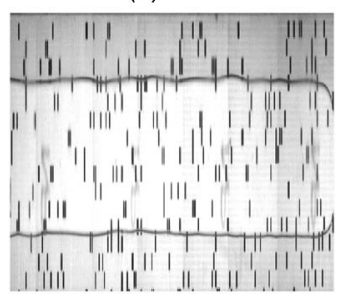

(b)

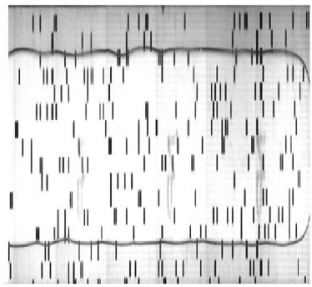

(e)

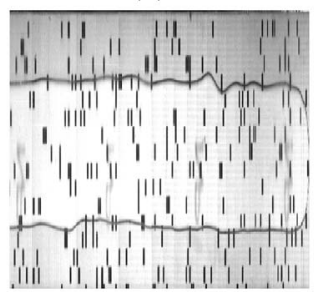

(c)

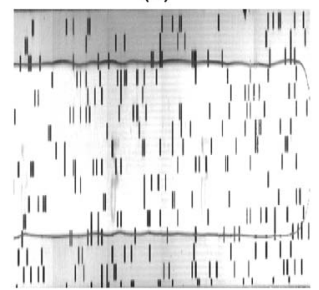

(f)

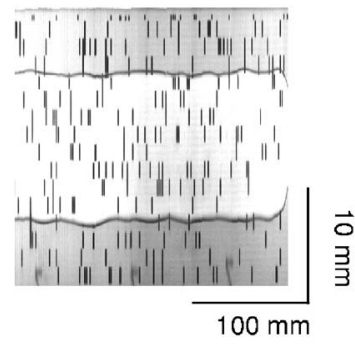

FIG. 6. Fingers in a cell with a $10 \%$ coverage and gap spacing $b=1.3 \mathrm{~mm}$ at several velocities. Corresponding capillary numbers are labeled with letters in Fig. 5. (A) $1 / B=18$, (B) $1 / B=88$, (C) $1 / B=198, \quad$ (D) $1 / B=339$, and $(\mathrm{E})$ $1 / B=1091$, (F) 1300 . 


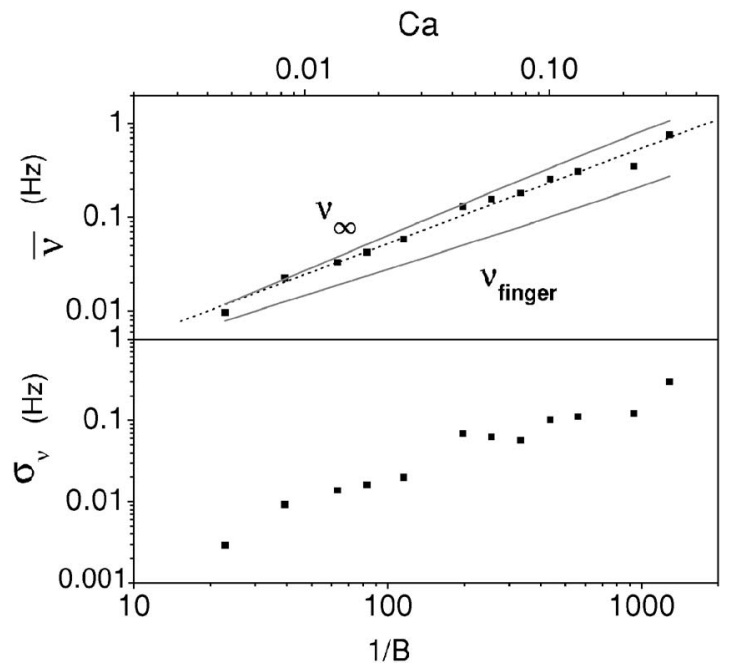

FIG. 8. Top, mean of the distribution of frequencies of the lateral instability vs $1 / B$. Squares are experimental values. Dotted line is the best fit to the same ones, $\nu_{\text {mean }}=(4.9 \pm 0.4)$ $\times 10^{-4}(1 / B)^{(1.02 \pm 0.04)} \mathrm{Hz}$. Bottom, standard deviation of the frequencies as a function of $1 / B$.

of the steady state which are always very close to each other since the width of the fingers is always greater than one-half of the channel width. The lateral fluctuations have characteristic length scales that are of the order of the channel width. The fact that there is a preferred frequency-or wavelength-indicates that the system is acting as a selective amplifier of static noise.

Comparison with numerical studies of the effect that temporal noise has on the Saffman-Taylor finger shape [14], leads to conclude that the effect of temporal noise and static noise are similar since both generate lateral fluctuations on the finger sides that undergo a process of selection and end up with similar frequency distributions. In our experiments, the distribution does not seem to be very sensitive to the intensity of static noise present in the system. Our experimental measurements indicate that for a given flow rate, rms fluctuations of the finger width, decrease with decreasing intensity of static noise. This might explain why the sides of the fingers are flat for typical Saffman-Taylor experiments despite the fact that any experiment has, inevitably, some static noise present in the cell. Also, since the wavelength of the instability is large, long channels are needed in order to observe it.

We have changed the capillary number by varying the flow rate. Distributions of frequencies indicate that the frequency that dominates the lateral fluctuation increases with increasing $\mathrm{Ca}$. This indicates that at large capillary numbers short wavelength fluctuations are more and more favorable. Also, the width of the distribution increases with increasing $\mathrm{Ca}$ indicating that the range of frequencies that grow increases with increasing $\mathrm{Ca}$.

The behavior of fluctuations of the finger width found in our experiments, is qualitatively similar to the one reported in Ref. [13], in the sense that, the magnitude of the width fluctuations decays as a power law of $\mathrm{Ca}$, at low $\mathrm{Ca}$, and increases for large values of $\mathrm{Ca}$. This similarity occurs, despite the fact that conditions for the experiments in Ref. [13] are very different from our experiments. First of all, their aspect ratios $W / b$ are large while ours are small. Second, they have made every effort to suppress spatial inhomogeneities in their cell, while we have intentionally introduced static disorder in it. However, the fact that our exponents are different, $-2 / 3$ in their case and $-1 / 3$ in our case, leaves open the question of the origin of the fluctuations observed in Ref. [13].

\section{ACKNOWLEDGMENTS}

We acknowledge technical support from A. Comerma and M. Quevedo. We acknowledge financial support of the $D i$ rección General de Investigación under Contract No. BFM2003-07749-C05-04. One of the authors (E. C. P.) acknowledges financial support from Generalitat de Catalunya and DGAPA, UNAM.
[1] M. Alava, M. Dube, and M. Rost, Adv. Phys. 53, 83 (2004).

[2] E. L. Decker, J. Ignés-Mullol, A. Baratt, and J. V. Maher, Phys. Rev. E 60, 1767 (1999).

[3] Y. Couder, in Perspectives in Fluid Dynamics, edited by G. K. Batchelor, H. K. Moffat, and M. G. Worster (Cambridge University Press, Cambridge, 2000), p. 53.

[4] C. Chevalier, M. Ben-Amar, D. Bonn, and A. Lindner, J. Fluid Mech. (to be published).

[5] L. Ristroph, M. Thrasher, M. B. Mineev-Weinstein, and H. Swinney, e-print nlin.PS0510049 (to be published).

[6] A. Dougherty, P. D. Kaplan, and J. P. Gollub, Phys. Rev. Lett. 58, 1652 (1987).

[7] A. Dougherty and J. P. Gollub, Phys. Rev. A 38, 3043 (1988).

[8] M. N. Barber, A. Barbieri, and J. S. Langer, Phys. Rev. A 36, 3340 (1987)

[9] R. Pieters, Phys. Rev. A 37, 3126 (1988).
[10] R. Pieters and J. S. Langer, Phys. Rev. Lett. 56, 1948 (1986)

[11] Y. Couder, N. Gérard, and M. Rabaud, Phys. Rev. A 34, R5175 (1986).

[12] M. Rabaud, Y. Couder, and N. Gerard, Phys. Rev. A 37, 935 (1988).

[13] M. G. Moore, A. Juel, J. M. Burgess, W. D. McCormick, and H. L. Swinney, Phys. Rev. E 65, 030601(R) (2002).

[14] R. Ledesma-Aguilar, M. Quevedo-Reyes, E. Corvera Poiré, and A. Hernández-Machado, Phys. Rev. E 71, 016312 (2005).

[15] A. Hernández-Machado, A. M. Lacasta, E. Mayoral, and E. Corvera Poiré, Phys. Rev. E 68, 046310 (2003).

[16] J. Soriano, J. Ortín, and A. Hernández-Machado, Phys. Rev. E 66, 031603 (2002).

[17] J. W. McLean and P. J. Saffman, J. Fluid Mech. 102, 455 (1981). 